\title{
O CUIDADOR DO IDOSO E SUA COMPREENSÃO SOBRE A PREVENÇÃO E O TRATAMENTO CIRÚRGICO DAS FRATU- RAS DE FÊMUR ${ }^{1}$
}

\author{
Carla Roberta Monteiro* \\ Ana Cristina Mancussi e Faro**
}

\section{Resumo}

Este estudo teve como objetivo conhecer a percepção do cuidador do idoso submetido à cirurgia ortopédica como opção de tratamento para fratura de fêmur, sobre o trauma, os cuidados no pós-operatório e prevenção. A pesquisa foi realizada no Instituto de Ortopedia e Traumatologia do Hospital das Clínicas da Faculdade de Medicina da Universidade de São Paulo, a coleta de dados se deu por meio da realização de entrevistas, tendo participado do estudo 15 cuidadores. Em 86 \% dos casos, a fratura fora ocasionada por queda, tendo ocorrido predominantemente em ambiente domiciliar. Quanto aos cuidados pós-operatórios, $60 \%$ dos cuidadores não citaram qualquer cuidado especial referente ao vestuário, enquanto apenas $20 \%$ referiram a deambulação sem carga. Quanto à prevenção das fraturas em idosos, apenas 26,66\% dos cuidadores citaram como medidas, ações simples como a retirada de tapetes e $13,33 \%$ deles acreditam que não há como prevenir as quedas e fraturas em idosos. Diante dos resultados, conclui-se que há a necessidade de repensar a assistência de enfermagem quanto ao planejamento da alta hospitalar.

Palavras-chave: Idoso. Cuidador. Fratura de Fêmur.

\footnotetext{
Pesquisa apresentada à Divisão de Enfermagem do Instituto de Ortopedia e Traumatologia do Hospital das Clínicas da Faculdade de Medicina da Universidade de São Paulo (IOT-HCFM-USP) como requisito parcial à obtenção do título de especialista em Enfermagem Ortopédica e Traumatológica. Pesquisa vinculada ao Grupo de Pesquisa: Reabilitação e Funcionalidade em Enfermagem Traumato-Ortopédica.

* Enfermeira. Pesquisadora Executante. E-mail: carlamonteiro@usp.br

** Enfermeira. Livre Docente do Departamento de Enfermagem Médico Cirúrgica da Escola de Enfermagem da USP. Orientadora da pesquisa. E-mail: rafacris@usp.br
} 


\section{Introdução}

As questões relativas à terceira idade têm crescido em importância ultimamente, uma vez que o envelhecimento da população é um fenômeno global, que traz importantes repercussões nos campos social e econômico, especialmente em países em desenvolvimento.

Com base no crescimento populacional de idosos, estima-se que esta faixa etária da população representará, em meados do século XXI, cerca de $40 \%$ das pessoas acometidas pelo trauma (SOUZA; IGLESIAS, 2003).

As alterações estruturais e funcionais, assim como a coexistência de doenças sistêmicas predispõem os idosos a diversos acidentes. A progressão da idade é acompanhada de mudanças previsíveis em praticamente todos os sistemas do organismo, merecendo destaque as alterações do sistema cardíaco, nervoso, sensorial e músculo-esquelético, uma vez que as quedas, principal causa de acidente e incapacidade na terceira idade, geralmente acontecem por anormalidades do equilíbrio, fraqueza muscular, desordens visuais, anormalidades do passo, doença cardiovascular, alteração cognitiva e consumo de medicamentos, além dos fatores extrínsecos, representados por circunstâncias sociais e ambientais que criam desafios aos idosos.

Um estudo relacionado à causas e conseqüências das quedas em idosos (FABRICIO; RODRIGUES; COSTA JÚNIOR, 2004) apontou as fraturas como sendo a conseqüência mais comum representando $64 \%$. Dentre elas, as mais freqüentes foram as de fêmur, representando $62 \%$ das fraturas.

A fratura de fêmur tem sido apontada como uma causa comum e importante de mortalidade e perda funcional entre os idosos (SAKAKI et al., 2004).

O tratamento da maioria das fraturas de fêmur é cirúrgico, sendo o conservador reservado somente para algumas fraturas incompletas ou sem desvio. O idoso tem sua reserva funcional diminuída e apresenta um número grande de doenças crônicas associadas, estando assim muito mais sujeito a complicações no pós-operatório tanto imediato quanto tardio.

No Brasil, o Estado se apresenta como um parceiro pontual com responsabilidades reduzidas, que atribuí à família a responsabilidade 
maior dos cuidados desenvolvidos em casa ao idoso com dependência.

Um estudo, sobre o suporte domiciliar aos adultos com perda de independência (CALDAS, 2003), aponta que mais de 90\% das famílias não receberam ajuda de serviços, organizações ou grupos voluntários e/ou agências particulares. Deste modo, constata-se que os cuidados oriundos de redes informais de apoio constituem a mais importante fonte de suporte a idosos, merecendo destaque a figura do cuidador.

Estudos indicam que os cuidadores apresentam necessidades que vão desde os aspectos materiais até os emocionais. No mesmo estudo, cuidadores mencionaram que, quando da alta hospitalar, os familiares raramente recebem informações claras a respeito da doença, orientação ou apoio para os cuidados. A maioria desses cuidadores foi orientada, superficialmente, sobre medicação, alimentação e retornos (CALDAS, 2003).

Caldas (2003) ressalta que as recentes tentativas brasileiras de montar programas de assistência domiciliar, mostram que os estudos sobre cuidados e sobre cuidadores tornam-se cada vez mais importantes e absolutamente necessários para subsidiar essas iniciativas. Além disso, no Brasil existem poucos estudos sobre as demandas do cuidador domiciliar do idoso, já que este personagem ainda é desconhecido no cenário público.

Diante do exposto e do fato de que há a necessidade de reconhecer que esta questão tende a se tornar um problema de Saúde Pública, surgiu a inquietação para a realização do presente estudo, a fim de serem levantadas as demandas dos cuidadores que posteriormente possam nortear treinamentos dirigidos a eles.

\section{Objetivos}

Caracterizar os cuidadores de idosos que sofreram fratura de fêmur quanto a idade, sexo, escolaridade, vínculo, parentesco, ocupação e profissão atuais; verificar o entendimento do cuidador quanto ao trauma ortopédico, cuidados no pós-operatório e prevenção de fraturas em idosos. 


\section{Método e Casuística}

Trata-se de um estudo exploratório e descritivo com abordagem quantitativa. A população do estudo foi composta pelos cuidadores de todos os idosos internados durante o período de dois meses na clínica de Geriatria do Instituto de Ortopedia e Traumatologia do Hospital das Clínicas da Faculdade de Medicina da Universidade de São Paulo, submetidos à cirurgia ortopédica por fratura de fêmur, perfazendo uma população de 15 cuidadores.

As entrevistas foram realizadas pela pesquisadora executante, em local apropriado e privativo nas imediações da unidade, conforme a disponibilidade referida pelo cuidador. É oportuno definir que, nesta pesquisa, entende-se por cuidador a pessoa familiar ou não que esteja acompanhando o idoso durante a internação.

O roteiro de entrevistas, na primeira parte é composto por dados coletados em prontuário relacionados aos idosos, ao trauma, diagnóstico e terapêutica e dados relativos aos cuidados de enfermagem pósoperatórios requeridos. A segunda parte visa a caracterização do cuidador do idoso através do levantamento de dados sócio-demográficos. $\mathrm{Na}$ terceira parte, foi investigado o entendimento dos cuidadores sobre a prevenção de fraturas em idosos, sobre o diagnóstico, tratamento, e cuidados pós-operatórios.

Cabe ressaltar que os sujeitos da pesquisa têm idade igual ou superior a 18 anos, e aceitaram participar do estudo após o esclarecimento e assinatura do termo de consentimento livre e esclarecido, garantindo seu sigilo, anonimato e livre participação.

\section{Resultados e Discussão}

Apresentamos a seguir os resultados obtidos com a aplicação do roteiro de entrevistas e uma breve discussão baseada na literatura mais recente.

\subsection{Caracterização dos Idosos e Cuidados Demandados}

Os idosos envolvidos no estudo têm em sua maioria entre 65 a 75 anos de idade, predominando o sexo feminino, dado condizente com 
outros estudos epidemiológios das fraturas do terço proximal do fêmur (ROCHA et al., 2003; PEREIRA et al., 1993).

O diagnóstico das fraturas de fêmur variou, predominando as fraturas transtrocanterianas, responsáveis por sete dos 15 casos atendidos. Em seguida temos a fratura de colo de fêmur com quatro casos.

A causa mais freqüente foi a queda, perfazendo $86 \%$ dos casos, seguida pelo atropelamento $(7 \%)$ e fratura iminente $(7 \%)$ o que demonstra que a maioria dessas lesões podem estar associadas às doenças prévias à época de fratura e o mais importante, que elas podem ser evitadas por procedimentos simples como a prevenção e tratamento das doenças de base, modificações de vida cotidiana e eliminação de obstáculos que eventualmente possam causar acidentes.

Algumas pesquisas esclarecem que as quedas mais freqüentes ocorrem nos próprios domicílios. Um estudo envolvendo uma população composta por pessoas com 60 anos ou mais, vítimas de quedas, de ambos os sexos, com residência fixa na cidade de Ribeirão Preto, São Paulo, atendidas em duas unidades de internação de um hospital universitário localizado no mesmo município, no ano de 2000 (FABRICIO; RODRIGUES; COSTA JÚNIOR, 2004), revelou que 54\% das quedas apresentaram como causa ambiente inadequado e que $66 \%$ das quedas ocorreram no próprio lar do idoso sendo que apenas $22 \%$ das quedas ocorreram na rua, sendo a conseqüência mais comum as fraturas, que foram evidenciadas em $64 \%$ dos casos.

Assim como o diagnóstico, o tratamento para as fraturas de fêmur variou, predominando o uso do parafuso dinâmico de quadril empregado nas fraturas transtrocanterianas, perfazendo sete casos, seguido da artroplastia parcial de quadril (20\%), correspondente às fraturas de colo de fêmur. Nestes tipos de fratura é muito importante optar por tratamentos que permitam a mobilização precoce dos pacientes, evitando-se os inconvenientes provocados pela permanência do idoso em repouso no leito, como infecções pulmonares, escaras ou problemas circulatórios.

Foram identificadas em prontuário médico, as doenças prévias à época de fratura, merecendo destaque as doenças cardiovasculares, aparecendo em dez dos 15 casos levantados, o que inclui hipertensão arterial, arritmias e insuficiência coronariana. O que sugere que este possa ser um fator intrínseco de significância na ocorrência de quedas 
dos idosos estudados. O tempo de internação hospitalar variou de seis a 22 dias, o que demonstra a heterogeneidade dos pacientes, havendo casos mais simples, sem intercorrências clínicas e outros mais graves do ponto de vista ortopédico e/ou clínico, prolongando o período de internação.

O período pós-operatório variou de um a dez dias, sendo que os cuidadores foram predominantemente abordados pela pesquisadora no terceiro dia de pós-operatório (52\%), pois buscou-se abordar os sujeitos da pesquisa na véspera da alta hospitalar, quando grande parte das informações a respeito dos cuidados pós-operatórios a serem demandados em domicílio deveriam ter sido fornecidas aos cuidadores.

Quanto aos cuidados pós-operatórios demandados pelos idosos em enfermaria: 11 idosos $(73,33 \%)$ requeriam a realização de banho no leito; $(80 \%)$ requeriam comadre ou papagaio às eliminações; sua totalidade necessitava de cuidados cutâneos de prevenção de úlceras por pressão, realização de curativo em ferida operatória, observação de alterações de circulação dos membros inferiores, observação de alterações da sensibilidade e motricidade do membro operado e exercícios de extensão e flexão de tornozelos. Do total de idosos, $46,66 \%$ deles demandavam atenção quanto à alterações da pressão arterial e outros $13,33 \%$ quanto à alterações da glicemia capilar. Ainda, $13,33 \%$ dos idosos deveriam ter suas grades do leito mantidas elevadas por quadro demencial.

O idoso pode apresentar elevado risco de complicações, pneumonia, tromboembolismo, úlceras por pressão, cálculo renal, impactação fecal e contraturas estão entre as principais complicações que uma especial atenção da enfermeira é capaz de prevenir.

Atividades dentro dos limites determinados pelo médico devem ser promovidas, incluindo exercícios de inspiração e expiração profunda e tosse, exercícios isométricos e freqüentes mudanças de posição. A ingesta de líquidos deve ser encorajada e as características da urina devem ser observadas. Uma boa nutrição irá promover a cura e a resistência à infecção e diminuir a ocorrência de outras complicações. Exercícios envolvendo as articulações e o correto posicionamento pode prevenir contraturas. $\mathrm{O}$ correto alinhamento corpóreo pode ser mantido com coxins e suportes. Manter a pele seca e limpa, prevenir pontos de pressão, estimular a circulação por meio de massagens e mudança 
freqüente de decúbito são ações que podem reduzir o risco de úlceras por pressão. Colchões especiais, almofadas de água e outros artifícios são benéficos, porém eles não substituem o cuidado adequado com a pele e as freqüentes mudanças de posição. Além do que já foi dito, é importante que o paciente seja mobilizado o mais cedo possível (ELIOPOULOS, 2001).

Segundo Macedo (2003) a mobilização passiva deve ser precoce, e nas 48 horas pós- operatório o paciente deverá sentar fora do leito. A deambulação depende do vigor físico do paciente, e o apoio com carga deve estar relacionado á confiabilidade da montagem, que depende da qualidade do osso e da técnica cirúrgica.

Leme e Carelli (1998) ainda enfatizam que a fisioterapia respiratória e motora são fundamentais, no intuito de evitar as complicações pulmonares, Trombose Venosa Profunda e outras complicações da imobilidade nesta faixa etária.

\subsection{Caracterização dos Sujeitos da Pesquisa}

Os cuidadores envolvidos na pesquisa tinham em sua maioria $33 \%$ de 40 a 50 anos de idade. Do total, $80 \%$ eram do sexo feminino, $40 \%$ eram casados e igualmente $40 \%$ eram solteiros.Eram cuidadores informais $86,60 \%$, sendo constituídos em sua maioria por filhos(as) dos idosos. Quanto à escolaridade percebe-se heterogeneidade, pois 33\% dos cuidadores têm nível Fundamental incompleto, seguido por 27\% deles que têm Ensino Superior completo. A ocupação predominante foi a Do Lar, enquanto as profissões variaram entre estudante, cuidadora, arquiteta, psicóloga, assistente social, cabelereira, costureira, doméstica e técnica de enfermagem. Nota-se a predominância de atividades autônomas que permitam uma maior disponibilidade para estar junto ao idoso.

Denomina-se cuidador informal o elemento da família do idoso ou a ele relacionado, sem necessária formação específica, que passa a ser o responsável pelo desenvolvimento de ações relativas às necessidades do idoso. Tais atividades são, geralmente, exercidas de forma contínua, com substituições deficitárias e com pouca ou, predominantemente nenhuma remuneração. Já o denominado cuidador formal compreende o elemento contratado pelo idoso e/ou sua família, para exercer ações 
cuidativas. Estabelece-se, nesse caso um vínculo empregatício exigindo-se que esses cuidadores formais sejam habilitados por formação específica. O mais adequado é que seja um profissional da enfermagem, um auxiliar, um técnico ou um enfermeiro e tenha realizado um curso de capacitação em Geronto-Geriatria ou cuidado ao idoso (SANTOS, 2001).

A tendência à redução dos investimentos nos setores de saúde reverte na ampliação das responsabilidades familiares pelos cuidados aos idosos dependentes e incapacitados, sendo assim, a família predomina como alternativa no sistema de suporte informal aos idosos. Tal achado pôde ser evidenciado no presente estudo, bem como em outros estudos envolvendo cuidadores de idosos (NUNES, 2002; KARSH, 2003). Sendo assim, concluímos que o cuidador familiar revelou-se o ator principal na dinâmica dos cuidados pessoais necessários às atividades de vida diária dos portadores de lesões que lhe tiraram a independência, assim como há predominância do papel da mulher como cuidadora. Por causas predominantemente culturais, no Brasil, essa ainda é uma atribuição esperada pela sociedade e, esse papel é visto como sendo natural, pois está inscrito socialmente no papel de mãe. Cuidar dos familiares idosos, é portanto mais um dos papéis que a mulher assume na esfera doméstica.

Dos cuidadores entrevistados, 53,33\% moram com o idoso sendo que $46,67 \%$ não moram com o idoso, o que nos remete ao fato de que ter acompanhante constante não é uma variável capaz de impedir isoladamente as quedas em idosos, outras medidas devem ser tomadas quanto á prevenção deste evento nos idosos.

\subsection{Compreensão dos Cuidadores quanto ao Trauma, Tratamento e Cuidados a serem Dispendidos no Domicílio durante o Período Pós-Operatório Tardio}

Quando questionados sobre o que havia acontecido com o idoso, apenas dez cuidadores responderam que os idosos haviam quebrado o fêmur. Quando questionados sobre como essa lesão teria acontecido, 11 deles relataram que a causa teria sido uma queda, sendo que esses acidentes teriam acontecido predominantemente no ambiente domiciliar (cozinha, banheiro ou quarto), o que demonstra mais uma vez a 
necessidade de medidas simples de adaptação da moradia do idoso na prevenção destas lesões. Do total, 33\% acreditavam que o tratamento empregado se resumia à colocação de pinos e $26 \%$ deles compreendiam apenas que o idoso havia sido submetido a um procedimento cirúrgico, sem ter conhecimento e esclarecimento de maiores detalhes.

Quando questionada a sua compreensão e esclarecimento sobre os cuidados a serem dispendidos ao idoso no domicílio durante o período de pós-operatório tardio: $73,33 \%$ deles reconheciam a necessidade do uso da cadeira higiênica e um deles relatou não ter conhecimento quanto aos cuidados referentes à higiene corporal. Ainda, $60 \%$ deles não citou qualquer cuidado especial referente ao vestuário, $40 \%$ deles citaram como necessidade, o uso do andador para o auxílio da deambulação, apenas 20\% referiram o entendimento da importância da deambulação sem carga enquanto que igualmente $20 \%$ deles acreditavam que não deveria haver deambulação no domicílio no período pósoperatório. Quanto aos cuidados referentes à incisão cirúrgica, 46,66\% compreendiam que a limpeza da ferida deveria ser realizada apenas com água e sabão durante o banho, ainda, 20\% deles acreditavam que a limpeza da ferida deveria ser realizada em casa igualmente ao método empregado no hospital, ou seja, limpar a ferida com solução fisiológica e cobri-lá com gaze estéril, sendo que $20 \%$ deles não citaram qualquer cuidado referente à ferida operatória e um deles referiu não saber como proceder quanto à incisão cirúrgica. Em relação à medicação, 26,66\% dos cuidadores citaram como cuidado principal respeitar os horários de administração e administrar criteriosamente os remédios para controle das doenças de base. Quanto às eliminações, $40 \%$ dos cuidadores reconheceram a necessidade de encaminhar os idosos ao banheiro em cadeira higiênica. Outros cuidados referidos pelos cuidadores incluem a realização de fisioterapia e alimentação saudável. 
TABELA 1 - Distribuição das respostas dos cuidadores dos idosos submetidos à cirurgia ortopédica, como opção de tratamento para fratura de fêmur, referente aos cuidados pós-operatórios a serem dispendidos no domicílio, São Paulo, IOT-HCFMUSP, 2005

\begin{tabular}{|c|c|c|}
\hline \multirow[t]{2}{*}{ Cuidados referentes à higiene corporal } & \multicolumn{2}{|c|}{ Distribuição } \\
\hline & $N$ & $\%$ \\
\hline Realizar banho em cadeira higiênica & 11 & 73,33 \\
\hline Realizar banho no leito & 3 & 20,00 \\
\hline Não sabe & 1 & 6,66 \\
\hline \multirow[t]{2}{*}{ Cuidados referentes ao vestuário } & \multicolumn{2}{|c|}{ Distribuição } \\
\hline & $N$ & $\%$ \\
\hline Usar saias ou vestidos & 3 & 20,00 \\
\hline Usar roupas confortáveis & 1 & 6,66 \\
\hline Usar camisola & 1 & 6,66 \\
\hline Não trajar vestuário na porção inferior & 1 & 6,66 \\
\hline Não citou & 9 & 60,00 \\
\hline Não sabe & 1 & 6,66 \\
\hline \multirow[t]{2}{*}{ Cuidados referentes à locomoção } & \multicolumn{2}{|c|}{ Distribuição } \\
\hline & N & $\%$ \\
\hline Deambular com auxílio de andador & 6 & 40,00 \\
\hline Deambular sem carga por 30 dias & 3 & 20,00 \\
\hline Não deve haver locomoção & 3 & 20,00 \\
\hline Utilizar cadeira de rodas & 2 & 13,33 \\
\hline Deambular com auxílio de bengala & 1 & 6,66 \\
\hline Não citou & 1 & 6,66 \\
\hline \multirow[t]{2}{*}{ Cuidados referentes à incisão cirúrgica } & \multicolumn{2}{|c|}{ Distribuição } \\
\hline & $N$ & $\%$ \\
\hline Limpar a ferida com água e sabão & 7 & 46,66 \\
\hline $\begin{array}{l}\text { Limpar a ferida com solução fisiológica e cobrir com } \\
\text { gaze estéril }\end{array}$ & 3 & 20,00 \\
\hline $\begin{array}{l}\text { Comparecer ao retorno médico para a retirada de } \\
\text { pontos }\end{array}$ & 1 & 6,66 \\
\hline Irrigar área doadora de enxerto de pele com óleo & 1 & 6,66 \\
\hline Realizar curativo conforme orientação do farmacêutico & 1 & 6,66 \\
\hline Realizar curativo em unidade básica de saúde & 1 & 6,66 \\
\hline
\end{tabular}




\begin{tabular}{|c|c|c|}
\hline Não citou & 3 & 20,00 \\
\hline Não sabe & 1 & 6,66 \\
\hline Cuidados referentes à medicação & \multicolumn{2}{|c|}{ Distribuição } \\
\hline & $N$ & $\%$ \\
\hline Respeitar os horários de administração & 4 & 26,66 \\
\hline $\begin{array}{l}\text { Administrar os remédios para controle de doenças de } \\
\text { base }\end{array}$ & 4 & 26,66 \\
\hline $\begin{array}{l}\text { Administrar remédios para controle de inflamação e } \\
\text { formação de trombos }\end{array}$ & 1 & 6,66 \\
\hline Administrar remédios para controle da dor & 1 & 6,66 \\
\hline Não esquecer de administrar as medicações & 1 & 6,66 \\
\hline Não citou & 4 & 26,66 \\
\hline Não sabe & 1 & 6,66 \\
\hline \multirow[t]{2}{*}{ Cuidados referentes às eliminações } & \multicolumn{2}{|c|}{ Distribuição } \\
\hline & $N$ & $\%$ \\
\hline Encaminhar ao banheiro em cadeira higiênica & 6 & 40,00 \\
\hline Manter fralda higiênica & 3 & 20,00 \\
\hline Oferecer comadre ou papagaio às eliminações & 3 & 20,00 \\
\hline Encaminhar ao vaso sanitário & 2 & 13,33 \\
\hline \multirow[t]{2}{*}{ Outros cuidados } & \multicolumn{2}{|c|}{ Distribuição } \\
\hline & $N$ & $\%$ \\
\hline Fisioterapia & 5 & 33,33 \\
\hline Providenciar alimentação saudável & 5 & 33,33 \\
\hline Providenciar segurança quanto a quedas & 1 & 6,66 \\
\hline Utilizar bolsa de água quente para alívio da dor & 1 & 6,66 \\
\hline Realizar massagem de conforto & 1 & 6,66 \\
\hline Comparecer aos retornos médicos & 1 & 6,66 \\
\hline $\begin{array}{l}\text { Improvisar arco de proteção para área doadora de } \\
\text { enxertia }\end{array}$ & 1 & 6,66 \\
\hline $\begin{array}{l}\text { Providenciar um farmacêutico para aplicação das } \\
\text { injeções }\end{array}$ & 1 & 6,66 \\
\hline Manter ambiente limpo & 1 & 6,66 \\
\hline Visita domiciliária & 1 & 6,66 \\
\hline Oferecer carinho e amor & 1 & 6,66 \\
\hline Não citou & 3 & 20,00 \\
\hline
\end{tabular}

Estud. interdiscip. envelhec., Porto Alegre, v. 10, p. 105-121, 2006. 
Questionamos aos cuidadores sobre informações recebidas referentes aos cuidados pós-operatórios a serem dispendidos no domicílio, $41 \%$ deles referiram não ter recebido esclarecimentos da equipe de saúde, e relataram que sua compreensão acerca dos cuidados a serem dispendidos eram advindos de experiências anteriores de internação ou então da observação dos cuidados dispendidos pelos profissionais da enfermagem durante todo o período de internação. Já 35\% deles referiram que a equipe de enfermagem foi a responsável pelo fornecimento das informações e esclarecimento de dúvidas. O que destaca o importante papel a ser desempenhado pelo enfermeiro junto à toda a equipe de enfermagem nesse processo de alta hospitalar do binômio idoso/cuidador.

\subsection{Compreensão quanto às Medidas de Prevenção de Fraturas em Idosos}

A presença de fratura e queda prévias como fator de risco para novas quedas foi descrita em alguns estudos prospectivos. A possibilidade de ter experimentado uma queda anterior com conseqüência séria, como fratura, parece imputar ao idoso uma maior vulnerabilidade a novos episódios de queda independentemente da freqüência deles.

Neto (2002), verificou que 54\% das quedas sofridas pelos idosos tiveram como causa ambiente inadequado tais como piso escorregadio $26 \%$, atrapalhar-se com objetos no chão $22 \%$, trombar em outras pessoas $11 \%$, subir em objetos para alcançar algo (7\%), queda da cama (7\%), problemas com degrau (7\%) e outros.

Quando indagamos os cuidadores acerca das medidas que poderiam ser empregadas para evitar as fraturas em idosos, foram citadas medidas relacionadas à moradia/ambiente; medidas relacionadas ao idoso e medidas relacionadas à família/acompanhante, destacando-se a retirada de tapetes $26,66 \%$; o não uso de escadas $26,66 \%$; o não enceramento do piso $20 \%$; a prevenção da osteoporose $20 \%$, a conscientização do idoso $20 \%$, o uso de calçados fechados $13,33 \%$ e a presença constante de um acompanhante 46,66\%. Dois dos cuidadores acreditam que a fratura de idosos é um acontecimento inevitável e que portanto não existem medidas capazes de prevenir estas lesões, ou seja, que a queda é inerente ao envelhecimento. 
TABELA 2 - Distribuição das respostas dos cuidadores dos idosos submetidos à cirurgia ortopédica, como opção de tratamento para fratura de fêmur, referente às medidas de prevenção de fraturas em idosos, São Paulo, IOT-HCFMUSP, 2005

\begin{tabular}{l|r|r}
\hline Medidas relacionadas à moradia/ & \multicolumn{2}{|c}{ Distribuição } \\
ambiente & $\ldots \ldots \ldots \ldots \ldots \ldots \ldots \ldots$ \\
\hline Retirar tapetes & $N$ & $\%$ \\
\hline Evitar uso de escadas & 4 & 26,66 \\
\hline Não encerar o chão & 3 & 26,66 \\
\hline Afastar móveis & 1 & 6,66 \\
\hline Instalar barras de auxílio & 1 & 6,66 \\
\hline Instalar corrimãos & 1 & 6,66 \\
\hline Equipar a cama com grades laterais & 1 & 6,66 \\
\hline Instalações e travessias públicas & 1 & 6,66 \\
adequadas & & \\
\hline \hline Medidas relacionadas ao idoso & Distribuição \\
\hline & $N$ & $\% \ldots$ \\
\hline Prevenir osteoporose & 3 & 20,00 \\
\hline Conscientizar o idoso & 3 & 20,00 \\
\hline Utilizar calçados fechados & 2 & 13,33 \\
\hline Realizar atividades vagarosamente & 1 & 6,66 \\
\hline Manter alimentação balanceada & 1 & 6,66 \\
\hline Vestir-se sentado & 1 & 6,66 \\
\hline \hline Medidas relacionadas à família/ & Distribuição \\
acompanhante & $\ldots \ldots \ldots \ldots \ldots \ldots \ldots \ldots \ldots$ \\
\hline Manter presença de acompanhante & 7 & 46,66 \\
\hline Assistir no banho & 2 & 13,33 \\
\hline Conscientizar o acompanhante & 1 & 6,66 \\
\hline Outras medidas & Distribuição \\
\hline Não há como prevenir & $N$ & $\% \ldots$ \\
\hline
\end{tabular}


Em 1999, ano internacional do idoso, a Sociedade Brasileira de Ortopedia e Traumatologia (SBOT) com a colaboração de profissionais da área de arquitetura, implantou o projeto "Casa Segura" (BARROS, 2000), com o intuito de tornar público e acessível as recomendações arquitetônicas para uma ambientação adequada que permita ao idoso viver em um ambiente seguro e confortável, garantindo-lhe uma maior independência, qualidade de vida e dignidade.

\section{Considerações Finais}

Os resultados obtidos com este estudo, vêm reforçar o fato de que os cuidadores ainda são predominantemente cuidadores informais, ou seja, indivíduos leigos na área da saúde, e que portanto necessitam de apoio de toda a equipe de saúde e informações sobre a realização de cuidados, bem como de orientações relativas à adaptação do ambiente ao idoso. $\mathrm{O}$ que nos faz refletir sobre a necessidade de repensarmos sobre a assistência de enfermagem no preparo para alta hospitalar.

Muitas vezes, a atuação dos profissionais de saúde, no preparo da alta, ocorre quando o paciente já está saindo do hospital. A orientação de alta do paciente deve ser planejada pela equipe de saúde desde a sua admissão para que não haja uma sobrecarga de informações no momento da saída do hospital. Neste momento, recomenda-se apenas reforçar algumas orientações dadas anteriormente.

Desta forma, sugerimos que devemos planejar a execução de um programa progressivo de orientação de alta, que vise preparar de forma eficiente o indivíduo e seu cuidador a exercer o cuidado após sua saída do hospital. Para tanto, há necessidade de que não só o indivíduo, mas também o cuidador possam além de observar, serem incentivados a praticar o cuidado e desta forma demonstrar independência nesta prática, ainda no decorrer da internação. Ou seja, é essencial que a equipe de saúde, em conjunto com o enfermeiro desenvolva um planejamento e/ou um treinamento que permita o fornecimento orientações e demonstrações dos cuidados necessários com o paciente, de forma que seja possível esclarecer as dúvidas dos cuidadores e certificar-se da incorporação das orientações dadas a eles.

Da mesma forma, não podemos nos esquecer que faz-se necessário que a família do idoso seja orientada não somente quanto aos cui- 
dados a serem dispendidos ao idoso quando em domicílio, mas também quanto à importância das adequações a serem realizadas no ambiente em que o idoso vive, a fim de evitar novos episódios de quedas, fraturas e outras complicações.

\title{
ELDER'S CAREGIVER AND THEIR COMPREHENSION ABOUT PREVENTION AND SURGICAL TREATMENT OF FEMUR FRACTURES
}

\begin{abstract}
This study had the objective of knowing the elder's caregiver's perception who was submited by an orthopedic surgery as a treatment option for femur fracture, about the trauma, the post-operative care and prevention. The research was realized at the Orthopedic and Traumatologic Institute of São Paulo's University Clinics Hospital, the datas were collected by interviews, 15 caregivers participated of the study, $86 \%$ of the cases of fractures were caused by falls that happened in their majority in the elder's home. About the post-operative care, $60 \%$ of the caregivers didn't mentioned any special care relating to dress while only $20 \%$ mentioned the locomotion without weight. About the prevention of the fracture in elders, only $26,66 \%$ of the caregivers mentioned simple actions as take out the carpets and $13,33 \%$ of them believe that there is no way to prevent falls and fractures in elders. Then, we conclude that it's vital that we think more carefully about the nursing care relating to the planning of the discharge from hospital.

Keywords: Elder. Caregiver. Femur Fracture.
\end{abstract}

\section{REFERÊNCIAS}

BARROS, Cybele F. Monteiro de. Casa Segura: uma arquitetura para a maturidade. Rio de Janeiro: Armazém Digital, 2000.

CALDAS, Célia Pereira. Envelhecimento com Dependência: responsabilidades e demandas da família. Cadernos de Saúde Pública, Rio de Janeiro, v. 19, n. 3, p. 733-781, 2003. 
ELIOPOULOS, Charlotte. Gerontological Nursing. Philadelphia: Lippincot, 2001.

FABRICIO, Suzele Cristina Coelho; RODRIGUES, Rosalina A. Partezani; COSTA JÚNIOR, Moacyr Lobo da. Causas e Conseqüências de Quedas em Idosos atendidos em Hospital Público. Revista de Saúde Pública, São Paulo, v. 38, n. 1, p. 93-99, 2004.

KARSH, Ursula Margarida. Idosos Dependentes: famílias e cuidadores. $\mathrm{Ca}$ dernos de Saúde Pública, Rio de Janeiro, v. 19, n. 3, p. 861-866, 2003.

LEME, Luiz Eugênio Garcez; CARELLI, Clara de Rosa. Peculiaridades do Pré e Pós-operatório dos Pacientes Idosos em Procedimentos Ortopédicos. Acta Ortopédica Brasileira, São Paulo, v. 6, n. 1, p. 37-43, 1998.

MACEDO, Carlos Alberto Souza. Fraturas e Luxações do Quadril no Adulto. In: SIZINIO, Hebert et al. Ortopedia e Traumatologia: princípios e prática. São Paulo: Artmed, 2003. P. 636-649.

NETO, Matheus Papaléo. Gerontologia: a velhice e o envelhecimento em visão globalizada. São Paulo: Atheneu, 2002.

NUNES, Ivany Aparecida. O Cuidar do Idoso com Trauma Ortopédico realizado pelo Cuidador Familiar no Domicílio. 2002. 82 p. Dissertação (Mestrado) - Escola de Enfermagem, Universidade de São Paulo, São Paulo, 2002.

PEREIRA, Gilberto José Cação et al. Estudo epidemiológico retrospectivo das fraturas do terço proximal do fêmur na região de Botucatu. Revista Brasileira de Ortopedia, Rio de Janeiro, v. 28, p. 504-510, 1993.

ROCHA, Murilo A. et al. Estudo Epidemiológico Retrospectivo das Fraturas do Fêmur Proximal tratados no Hospital Escola da Faculdade de Medicina do Triângulo Mineiro. Revista Brasileira de Ortopedia, Rio de Janeiro, v. 36, n. 8, p. 311-316, 2003.

SAKAKI, Marcos H. et al. Estudo da Mortalidade na Fratura do Fêmur Proximal em Idosos. Acta Ortopédica Brasileira, São Paulo, v. 12, n. 4, p. 242-249, 2004. 
SANTOS, Silvana Sidney Costa. Enfermagem Gerontogeriátrica: reflexão à ação cuidativa. São Paulo: Robe, 2001

SOUZA, José Antonio Gomes de; IGLESIAS, Antonio Carlos R. G. Trauma no Idoso. Revista da Associação Médica Brasileira, São Paulo, v. 1, n. 48, p. 79-86, 2003.

Recebido: 11/12/2006 